\title{
Reduced Plasma Glucose by Asparagine Synthetase Knockdown in the Mouse Liver
}

\author{
Shinya Iida, ${ }^{a}$ Hiroyuki Kamiya, ${ }^{*}, a, b$ Akihiro Nakaya ${ }^{c}$ Yasuhiro Hayashi, ${ }^{a}$ Akihiro Sawada, ${ }^{d, e}$ \\ Noritada Kaji, ${ }^{f}$ Yoshinobu Baba, ${ }^{f}$ and Hideyoshi Harashima ${ }^{a}$ \\ ${ }^{a}$ Faculty of Pharmaceutical Sciences, Hokkaido University; Sapporo 060-0812, Japan: ${ }^{b}$ Graduate School \\ of Science and Engineering, Ehime University; 2-5 Bunkyo-cho, Matsuyama 790-8577, Japan: ${ }^{c}$ Center for \\ Transdisciplinary Research, Niigata University; Niigata 951-8585, Japan: ${ }^{d}$ Department of Applied Chemistry, \\ Graduate School of Engineering, Nagoya University; ${ }^{f}$ Department of Applied Chemistry, Graduate School of \\ Engineering, FIRST Research Center for Nanobiodevices, Nagoya University; Nagoya 464-8603, Japan: and \\ ${ }^{e}$ Department of Molecular and Pharmaceutical Biotechnology, Graduate School of Pharmaceutical Sciences, \\ The University of Tokushima; Shomachi, Tokushima 770-8505, Japan. \\ Received May 28, 2013; accepted September 30, 2013
}

Expression of the asparagine synthetase gene is dependent on extracellular glucose concentration. This gene was knocked-down in livers of male Balb/c mice by a hydrodynamic tail vein injection of small interfering RNA (siRNA) against the gene. This knockdown resulted in a significant decrease in plasma glucose concentration. These results suggested that asparagine synthetase is a novel protein that regulates plasma glucose levels.

Key words asparagine synthetase; small interfering RNA; plasma glucose

Type 2 diabetes mellitus (T2DM) is a multi-factorial disorder caused by genetic and environmental factors, such as obesity, physical inactivity, and an unhealthful diet. ${ }^{1,2)}$ The therapy for T2DM involves controlling blood glucose levels, which, in turn, prevents the development of complications such as microangiopathy, arteriosclerosis and peripheral neuropathy. Continuous high blood glucose levels are responsible for macrovascular and microvascular diseases as a consequence of accelerated atherogenesis.. ${ }^{3}$ Thus, an understanding of the molecular mechanisms associated with the onset of T2DM would be highly desirable, in terms of establishing fundamental therapies for the disease.

To find a novel protein that regulates plasma glucose levels, we analyzed the gene expression in the liver of KK-A ${ }^{y} / \mathrm{Ta} . J \mathrm{Jl}$ mice, a model for T2DM mice, before the onset of the disease using an oligo DNA microarray. We noticed that the asparagine synthetase $(A s n s)$ gene was included in the genes which expressions were altered in the liver (elevated in $\mathrm{KK}^{-} \mathrm{A}^{y} / \mathrm{Ta} . \mathrm{Jcl}$ mice, Iida et al., unpublished results). This gene was interesting since incubation of human HepG2 cells in glucose-free medium increases ASNS mRNA and extracellular glucose suppresses the expression in a concentration-dependent manner. ${ }^{4)}$

In this study, we delivered small interfering RNA (siRNA) against the Asns gene into livers of the mice by the hydrodynamics-based injection. The knockdown resulted in reduced plasma glucose concentrations. These results suggest that Asns is involved in the regulation of plasma glucose levels.

\section{MATERIALS AND METHODS}

Oligonucleotides Oligodeoxyribonucleotides used as polymerase chain reaction (PCR) primers and oligoribonucleotides used as siRNAs were obtained from sigma Genosys Japan (Ishikari, Japan) and Greiner Bio-One (Frickenhausen, Germany), respectively, in purified forms.

The authors declare no conflict of interest.
Animals Four week-old male Balb/c mice (Hokudo, Sapporo, Japan) were housed with free access to water and food at $22^{\circ} \mathrm{C}$ and $58 \%$ humidity with light from 7:00 to 19:00. All animal protocols were approved by the Institutional Animal Care and Research Advisory Committee of the Faculty of Pharmaceutical Sciences, Hokkaido University, Sapporo, Japan.

In Vivo Knockdown of Asparagine Synthetase by Hydrodynamics-Based Injection The hydrodynamics-based injection of the siRNA against ASNS was performed according to the method of Liu et al. ${ }^{5)}$ and Zhang et al. ${ }^{6)}$ siRNA $(150 \mu \mathrm{g}$ in $2 \mathrm{~mL}$ of phosphate-buffered saline) was injected into the tail vein of male four week-old Balb/c mice within $5 \mathrm{~s}$. The following siRNA was used: Asns sense, 5'-GGCUUA CUU AGGCAU GAA AdTdT-3' and Asns antisense, 5'-UUU CAUGCCUAA GUA AGCCdTdT-3'. At $48 \mathrm{~h}$ after injection, blood glucose levels were measured.

Reverse Transcription-Quantitative PCR (RT-qPCR) Total RNA was isolated from liver and cDNA was prepared from $500 \mathrm{ng}$ of total RNA as described previously. ${ }^{7)}$ The resulting cDNA was analyzed by quantitative PCR using an Applied Biosystems 7500 Real Time PCR System (Life Technologies, Carlsbad, CA, U.S.A.) and SYBR Green Realtime PCR Master Mix (Toyobo, Osaka, Japan) according to the manufacturer's instructions. The target regions of the Asns and glyceraldehyde-3-phosphate dehydrogenase (Gapdh) genes were amplified with following set of primers: ASNS forward primer 5'-dGGA TTGGCTGCC TTT TATCA-3' and ASNS reverse primer 5'-dAACTTGGGCCTCCTTGAG TT-3', GAPDH forward primer 5'-dCCACCC AGA AGACTG TGGAT-3' and GAPDH reverse primer 5'-dGGA TGC AGG GATGATGTTCT-3'. All samples were run in triplicate and data were calculated using the comparative threshold cycle method and are expressed as a ratio to GAPDH as a reference.

\section{RESULTS AND DISCUSSION}

We selected the Asns gene as a knockdown target. As- 
paragine synthetase catalyzes the reversible conversion of L-asparatate to L-asparagine. Although no studies have reported that asparagine synthetase is related to T2DM, this enzyme is an interesting target, since expression of the Asns gene is dependent on extracellular glucose concentration. ${ }^{4)}$

The siRNAs against ASNS were screened by knockdown efficiencies in mouse hepatoma Hepa 1-6 cells (data not shown). In this study, we delivered siRNAs into mouse livers by hydrodynamic tail vein injection. ${ }^{5,6,8)}$ This method enables the delivery of nucleic acids into liver cells without the use of any vehicle. It has been shown that $c a .40 \%$ of mouse liver cells express $\beta$-galactosidase when plasmid DNA containing the gene for this enzyme was administered using this method. Thus, it is likely that the siRNAs could be delivered to at least ca. $40 \%$ of liver cells. Serum transaminases (alanine aminotransferase (ALT) and aspartate aminotransferase (AST)), interleukin-6, and interferon $\beta$ were not affected by this administration method at $48 \mathrm{~h}$ after injection (data not shown), although the hydrodynamics-based method induces hepatic damage. In addition, the hydrodynamic injection did not alter the expression of the gene for endogenous phosphoenolpyruvate carboxykinase (PCK1), the rate-controlling enzyme in gluconeogenesis, in the liver.

The siRNA ( $150 \mu \mathrm{g}$ per mouse) against the ASNS mRNA was administered to Balb/c mice, and knockdown efficiencies were examined. siRNA against the green fluorescent protein (GFP) was used as a negative control. Gómez-Valadés et al. observed that knockdown of PCK1 in mice lowered blood glucose. ${ }^{9)}$ Anti-PCK1 siRNA was used as a positive control based on their report. The administration of anti GFP siRNA had no effect on ASNS mRNA level (data not shown). Blood glucose level was slightly increased after administration of the anti GFP siRNA when compared with that of untreated mice $(120 \mathrm{mg} / \mathrm{dL})$. This would be due to the hydrodynamic injection since blood glucose was $136 \mathrm{mg} / \mathrm{dL}$ when phosphate-buffered saline was injected.

When siRNA against the Asns gene was injected, the amount of ASNS mRNA in the liver was decreased significantly, indicating effective knockdown (Fig. 1). We measured plasma glucose concentrations in Balb/c mice at $48 \mathrm{~h}$ after the injection of siRNA against ASNS. We found that the administration resulted in a reduction in plasma glucose levels (Fig. 2). It thus appears that ASNS is involved in the regulation of plasma glucose concentrations in mice. Meanwhile, blood glucose was also decreased upon anti-PCK1 siRNA injection (the positive control) as expected. The amount of PCK1 mRNA was $78 \%$ of the anti-GFP siRNA group probably due to PCK1 mRNA abundance, which would reflect importance of PCK1 as a gluconeogenic enzyme.

In this study, the knockdown of the Asns gene in liver reduced plasma glucose concentrations in mice. In HepG2 cells, the expression of the Asns gene increases by decrease of glucose in medium and is promoted by activating transcription factor 3 (ATF3), which is up-regulated by the reduction of glucose levels, ${ }^{4,10)}$ suggesting that asparagine synthetase plays a direct role in glucose metabolism. This enzyme catalyzes the conversion of L-asparatate, which is synthesized from oxaloacetate in the citric acid cycle. Thus, the suppression of asparagine synthetase could affect glucose degradation. However, further studies are necessary with regard to this possibility.

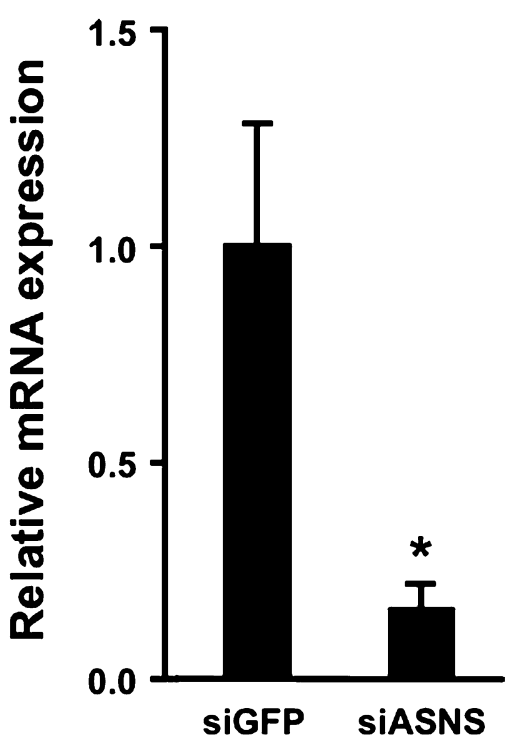

Fig. 1. Knockdown of the Asns Gene in Liver of Balb/c Mice

siRNA ( $150 \mu \mathrm{g}$ per mouse) against ASNS was administered by the hydrodynamics-based method. The amount of ASNS mRNA was measured by RT-qPCR at $48 \mathrm{~h}$ after siRNA introduction. The amount of mRNA was normalized relative to the mouse GAPDH mRNA contained in each sample. Values relative to that in mice treated with the control (GFP) siRNA are shown. Data are expressed as the mean+S.E.M. $(n=3) .{ }^{*} p<0.05 v s$. control siRNA.

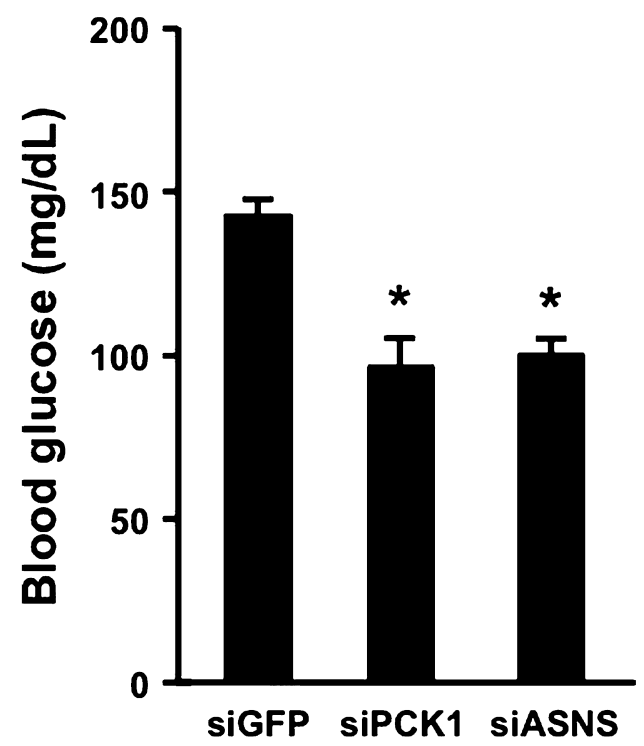

Fig. 2. Decreased Plasma Glucose by Injection of siRNA against ASNS siRNA ( $150 \mu \mathrm{g}$ per mouse) against ASNS was administered by the hydrodynamics-based method. Plasma glucose concentrations at $48 \mathrm{~h}$ after siRNA introduction in Balb/c mice under fasting condition were measured. siRNAs against GFP and phosphoenolpyruvate carboxykinase $(\mathrm{PCK} 1)^{9)}$ were used as negative and positive controls, respectively. Data are expressed as the mean+S.E.M. $(n=3)$. ${ }^{*} p<0.05$ vs. GFP siRNA.

We previously reported the genes expression of which is altered in T2DM-related organs in Otsuka Long-Evans Tokushima Fatty rats, another model for T2DM animals, before and after the onset of T2DM. ${ }^{7,11-13)}$ It would be interesting to examine whether change in expression is a cause or result of (pre-) diabetic state, for each gene. Detailed analyses of these genes, as well as the Asns gene, would help us understand the molecular mechanisms associated with the onset of T2DM, develop diagnostic tools for T2DM before onset, and establish fundamental therapies for the disease. 
Acknowledgments This work was supported by Grantsin-Aid for Scientific Research (A) from the Ministry of Education, Culture, Sports, Science and Technology of Japan, and by a Grant-in-Aid for Exploratory Research from the Japan Society for the Promotion of Sciences (JSPS).

\section{REFERENCES}

1) Eckel RH, Grundy SM, Zimmet PZ. The metabolic syndrome. Lancet, 365, 1415-1428 (2005).

2) Tusié Luna MT. Genes and type 2 diabetes mellitus. Arch. Med. Res., 36, 210-222 (2005).

3) Stumvoll M, Goldstein BJ, van Haeften TW. Type 2 diabetes: principles of pathogenesis and therapy. Lancet, 365, 1333-1346 (2005).

4) Barbosa-Tessmann IP, Pineda VL, Nick HS, Schuster SM, Kilberg MS. Transcriptional regulation of the human asparagine synthetase gene by carbohydrate availability. Biochem. J., 339, 151-158 (1999).

5) Liu F, Song YK, Liu D. Hydrodynamics-based transfection in animals by systemic administration of plasmid DNA. Gene Ther., $\mathbf{6}$, 1258-1266 (1999).

6) Zhang G, Budker V, Wolff JA. High levels of foreign gene expression in hepatocytes after tail vein injections of naked plasmid DNA. Hum. Gene Ther., 10, 1735-1737 (1999).

7) Iida S, Sato Y, Nakaya A, Shinohara Y, Hayashi Y, Sawada A, Nagata H, Kaji N, Kamiya H, Baba Y, Harashima H. Genome wide expression analysis of white blood cells and liver of pre-diabetic Otsuka Long-Evans Tokushima Fatty (OLETF) rats using a cDNA
Microarray. Biol. Pharm. Bull., 29, 2451-2459 (2006).

8) McCaffrey AP, Meuse L, Pham TTT, Conklin DS, Hannon GJ, Kay MA. Gene expression: RNA interference in adult mice. Nature, $\mathbf{4 1 8}$, 38-39 (2002).

9) Gómez-Valadés AG, Vidal-Alabró A, Molas M, Boada J, Bermúdez J, Bartrons R, Perales JC. Overcoming diabetes-induced hyperglycemia through inhibition of hepatic phosphoenolpyruvate carboxykinase (GTP) with RNAi. Mol. Ther., 13, 401-410 (2006).

10) Pan Y, Chen H, Siu F, Kilberg MS. Amino acid deprivation and endoplasmic reticulum stress induce expression of multiple activating transcription factor-3 mRNA species that, when overexpressed in HepG2 cells, modulate transcription by the human asparagine synthetase promoter. J. Biol. Chem., 278, 38402-38412 (2003).

11) Sato Y, Kuwajima M, Kamiya H, Harashima H. Calpain 10 as a predictive gene for type 2 diabetes: evidence from a novel screening system using white blood cells of Otsuka Long-Evans Tokushima Fatty (OLETF) rats. Biol. Pharm. Bull., 26, 1765-1768 (2003).

12) Hayashi Y, Iida S, Sato Y, Nakaya A, Sawada A, Kaji N, Kamiya H, Baba Y, Harashima H. DNA microarray analysis of type 2 diabetesrelated genes co-regulated between white blood cells and livers of diabetic Otsuka Long-Evans Tokushima Fatty (OLETF) rats. Biol. Pharm. Bull., 30, 763-771 (2007).

13) Hayashi Y, Kajimoto K, Iida S, Sato Y, Mizufune S, Kaji N, Kamiya $\mathrm{H}$, Baba Y, Harashima H. DNA microarray analysis of white blood cells and insulin-sensitive tissues reveals the usefulness of blood RNA profiling as a source of markers for predicting type 2 diabetes. Biol. Pharm. Bull., 33, 1033-1042 (2010). 